\title{
Distribution of the Bari-I transposable element in stable hybrid strains between Drosophila melanogaster and Drosophila simulans and in Brazilian populations of these species
}

\author{
Marcos de Lucca Jr. ${ }^{1}$, Claudia Marcia Aparecida Carareto ${ }^{1}$ and Carlos Roberto Ceron ${ }^{2}$ \\ ${ }^{1}$ Departamento de Biologia, Instituto de Biociências, Letras e Ciências Exatas, \\ Universidade Estadual Paulista, São José do Rio Preto, SP, Brazil. \\ ${ }^{2}$ Departamento de Química e Ciências Ambientais, Instituto de Biociências, Letras e Ciências Exatas, \\ Universidade Estadual Paulista, São José do Rio Preto, SP, Brazil.
}

\begin{abstract}
We analyzed the distribution of the Bari-I transposable element in Drosophila melanogaster (IN(1)AB), its sibling species Drosophila simulans (C167.4) and in eight hybrid strains derived from initial crosses involving $D$. simulans females and $D$. melanogaster males of the above cited strains as well as in Brazilian populations of these species. Polymerase chain reaction (PCR) data showed the presence of the Bari-I element among species populations and hybrid strains. Hybridization with a $703 \mathrm{bp}$ probe homologous to the Bari-l sequence showed that the number of Bari-I copies in D. melanogaster IN(1)AB was higher than in D. simulans $C 167.4$ strains. Hybrid strains presented Bari-I sequences related to both parental species. In addition some strains displayed a Bari-I sequence that came from $D$. melanogaster, suggesting introgression of $D$. melanogaster genetic material in the background of $D$. simulans. In contrast, some hybrids showed deletions of $D$. simulans Bari-I sequences.
\end{abstract}

Key words: Bari-I, Drosophila melanogaster, D. simulans, introgressive hybridization, transposable element.

Received: June 29, 2006; Accepted: January 31, 2007.

\section{Introduction}

The Bari-I element, initially found in Drosophila melanogaster, is a Class II transposable element with an open reading frame (ORF) able to encode for a polypeptide with 339 amino acids. The amino acids sequence of the putative protein in Bari-I is similar to the transposase of the Tc-1 element of Caernorhabditis elegans (Rosenzweig et al., 1983). While many heterochromatic elements exhibit rearrangements that result in a loss of coding capacity (O'Hare and Rubin, 1983; Di Nocera et al., 1986; Streck et al., 1986; Vaury et al., 1989; Crozatier et al., 1988; ) the Bari-I element presents heterochromatic and euchromatic copies with very similar ORFs in structure and sequence. Moreover, all euchromatic copies of Bari-I are homogeneous in both size and sequence, suggesting that this element is in its active form (Caizzi et al., 1993).

Studies regarding variability in the distribution of the Bari-I element conducted by Caggese et al. (1995) showed

Send correspondence to Carlos Roberto Ceron. Departamento de Química e Ciências Ambientais, Instituto de Biociências, Letras e Ciências Exatas, Universidade Estadual Paulista, Rua Cristóvão Colombo 2265, Jardim Nazareth, 15054-000 São José do Rio Preto, SP, Brazil. E-mail: ceron @ ibilce.unesp.br. that this element occurs in D. melanogaster and Drosophila simulans populations. However, differences occur in the distribution patterns of the Bari-I element between these two sibling Drosophila species, the main differences being that $D$. melanogaster contains heterochromatic groups of the Bari- $I$ element arranged in tandem on the second chromosome whereas in $D$. simulans there are just a few diffuse copies of this element spread throughout the genome.

Crosses between $D$. melanogaster and D. simulans occurs easily in laboratory (Sturtevant, 1920; Carracedo et al., 1998) and in nature (Sperlich, 1962; Tracey et al., 1973; Mensua and Perez, 1977; Kamping and Van Delden, 1988), but the offspring from these crosses frequently generate partially fertile offspring. Hybridization between these two species is the most studied system in the genus Drosophila, mainly in respect to the sterility observed in the hybrids the consequence of which is inhibited gene flow. Some studies have shown that crosses involving $D$. simulans females and D. melanogaster males can produce fertile descendants in some situations. For example, fertile hybrids females were observed in crosses between female $D$. simulans of strains C167.4, Oxnard and vermilion and male D. melanogaster of strains IN(1)AB or IN(1),f. (Davis et al.,1996), provid- 
ing a means to investigate the transference of DNA sequences between these two species.

We obtained stable hybrid strains from interspecific crossings between $D$. simulans $\mathrm{C} 167.4$ females and $D$. melanogaster IN(1)AB males. These strains contain sequences of the D. melanogaster copia retroelement introgressed into the genome of $D$. simulans (Ceron, Lisch and Kidwell, in preparation).

The study described in our present paper was undertaken to verify the presence and the distribution of the Bari-I element in D. melanogaster IN(1)AB and D. simulans C167.4 and analyze the distribution of this element in stable hybrids strains produced from crosses between these parental species. These analyses could help clarify the distribution of Bari-I in parental species as well as in hybrids. We also carried out a survey of the distribution of the Bari-I element in six D. melanogaster and five $D$. simulans populations collected at various locations throughout Brazil.

\section{Material and Methods}

\section{Fruitfly stocks}

The D. melanogaster $\mathrm{IN}(1) \mathrm{AB}$ and $D$. simulans C167.4 strains used were derived from the stocks of Margaret G. Kidwell's laboratory at the University of Arizona, USA. Eight stable hybrid strains obtained from initial crossings between $D$. simulans females and $D$. melanogaster males of the above cited strains were also investigated. We cross-mated $D$. simulans C167.4 females with $D$. melanogaster IN(1)AB males, some parental females producing viable $\mathrm{F}_{1}$ larvae which resulted in sterile males and fertile hybrid females. The hybrid females were individu- ally retrocrossed with $D$. simulans males (crossing in the opposite direction were not viable) and just one produced fertile female $F_{2}$ offspring and, once again, sterile males. The $\mathrm{F}_{2}$ females were retrocrossed once more with $D$. simulans males, this time producing $\mathrm{F}_{3}$ fertile males and females, crosses between which produced all the hybrid strains used in this study. These strains were approximately in the $80^{\text {th }}$ generation from the initial parental crosses. We also used D. melanogaster and D. simulans strains of collected from several Brazilian localities (Table 1). Stocks were kept on banana-agar medium in the Institute of Biosciences, Letters and Exact Sciences, São Paulo State University (Instituto de Biociências, Letras e Ciências Exatas, Universidade Estadual Paulista (UNESP)), São José do Rio Preto, São Paulo, Brazil.

\section{PCR and Southern blot analysis}

We extracted DNA from about 50 flies using the chloroform phenol method (Jowett, 1986). The PCR reactions for amplification of the Bari-I sequences were performed in a final volume of $25 \mu \mathrm{L}$ in $1 \mathrm{X}$ PCR buffer, containing 1 unit of Taq DNA polymerase (GIBCO), approximately $50 \mathrm{ng}$ of DNA template, $5 \mathrm{pmol} / \mu \mathrm{L}$ of each primer, $800 \mu \mathrm{M}$ dNTPs and $5 \mathrm{mM} \mathrm{MgCl} 2$. Amplification used 35 reaction cycles, each cycle consisting of 1 min denaturation at $95{ }^{\circ} \mathrm{C}, 1 \mathrm{~min}$ annealing at $60{ }^{\circ} \mathrm{C}$ and $1 \mathrm{~min}$ elongation at $72{ }^{\circ} \mathrm{C}$, followed by $5 \mathrm{~min}$ final extension at $72{ }^{\circ} \mathrm{C}$. Two sets of oligonucleotide primers were designed, one amplifying the $1.7 \mathrm{~kb}$ complete sequence of the Bari-I element (sense 5'TTGTGAAAATACTTTTGCACACCT CTGT3', antisense 5'ACAGAGGTGGTCAAAAGTATT TTCACAA3') and another amplifying an internal $703 \mathrm{bp}$ fragment complementary the positions 453 to 1196 of the

Table 1 - Brazilian Drosophila strains used in this study, geographic origin, latitude and year of collection.

\begin{tabular}{lllrl}
\hline Code & Geographic origin (town, state) & Latitude & Year collected & Collector, institution, state* \\
\hline $\begin{array}{llll}\text { D. melanogaster } \\
\text { TE }\end{array}$ & Teresina, Piauí (PI) & $2^{\circ} 32^{\prime} \mathrm{S}$ & 1996 & Z.M. Silva, UEPI, PI \\
SL & São Luis, Maranhão (MA) & $5^{\circ} 09^{\prime} \mathrm{S}$ & 1995 & S.R.P. Martins, UFMA, MA \\
SR & Santana do Riacho, Minas Gerais (MG) & $18^{\circ} 20^{\prime} \mathrm{S}$ & 1995 & C.R. Vilela, USP, SP \\
NH & Novo Horizonte, São Paulo (SP) & $21^{\circ} 29^{\prime} \mathrm{S}$ & 1999 & F.R. Torres, UNESP, SP \\
MA & Maringá, Paraná (PR) & $23^{\circ} 25^{\prime} \mathrm{S}$ & 1996 & A.S. Lapenta, UEM, PR \\
SM & Santa Maria, Rio Grande do Sul (RS) & $30^{\circ} 02^{\prime} \mathrm{S}$ & 1995 & V.L.S.V. Gaiesky, UFRGS, RS \\
\hline D. simulans & & & & \\
MN & Manaus, Amazonas (AM) & $3^{\circ} 07^{\prime} \mathrm{S}$ & 1997 & I.R. Cabral, UFPA, PA \\
GO & Goiânia, Goiás (GO) & $16^{\circ} 40^{\prime} \mathrm{S}$ & 1988 & D. C. Rigue, UNESP, SP \\
NH & Novo Horizonte, SP & $21^{\circ} 29^{\prime} \mathrm{S}$ & 1999 & F. R. Torres, UNESP, SP \\
MA & Maringá, PR & $23^{\circ} 25^{\prime} \mathrm{S}$ & 1996 & A.S. Lapenta, UEM, PR \\
BG & Bento Gonçalves, RS & $29^{\circ} 10^{\prime} \mathrm{S}$ & 1995 & V.L.S.V. Gaiesky, UFRGS, RS \\
\hline
\end{tabular}

*All institutions were in Brazil. Key to abbreviations: UEPI = Universidade Estadual do Piaú́; UFMA = Universidade Federal do Maranhão; USP = Universidade de São Paulo; UNESP = Universidade Estadual Paulista; UEM = Universidade Estadual de Maringá; UFRGS = Universidade Federal do Rio Grande do Sul ; UFPA = Universidade Federal do Pará. 
Bari-I element (sense 5'ATTCGTCGCAGGCTAAAAG A3', antisense 5'TTGTAACACCACCTTTGGCA3').

To estimate the Bari-I copy number we submitted Bari-I genomic DNA to overnight digestion with the Hind III endonuclease (GIBCO), for which there is only one cleavage in Bari-I sequence. DNA fragments were separated by electrophoresis on $1 \%(\mathrm{w} / \mathrm{v})$ agarose gels and transferred to nylon membranes (Hybond N+/AmershamPharmacia). Hybridization was performed using the ECL Direct Nucleic Acid Labeling and Detection Systems chemoluminescent hybridization system (Amersham Life Sciences) using $42{ }^{\circ} \mathrm{C}$ as the hybridization and washing temperatures. The probe used was the $D$. melanogaster Bari-I element $703 \mathrm{bp}$ internal fragment cloned into the pUC8 plasmid (provided by R. Caizzi Bari University, Bari, Italy).

\section{Results}

\section{Identification of the Bari-I element}

The PCR analyses verified the presence of the Bari-I element in D. melanogaster $\mathrm{IN}(1) \mathrm{AB}$ and $D$. simulans C167.4 using the primers described above which amplify the complete $1.7 \mathrm{~kb}$ sequence of the Bari-I element (Figure 1 ), but these primers produced only very faint amplified bands in the hybrids strains. However, the primers for the 703 bp Bari-I element internal fragment produced bands with better definition in all parental and hybrids strains (Figure 2).

\section{The Bari-I copy numbers}

The number of Bari-I copies in D. melanogaster IN(1)AB, D. simulans C167.4 and eight derived hybrid

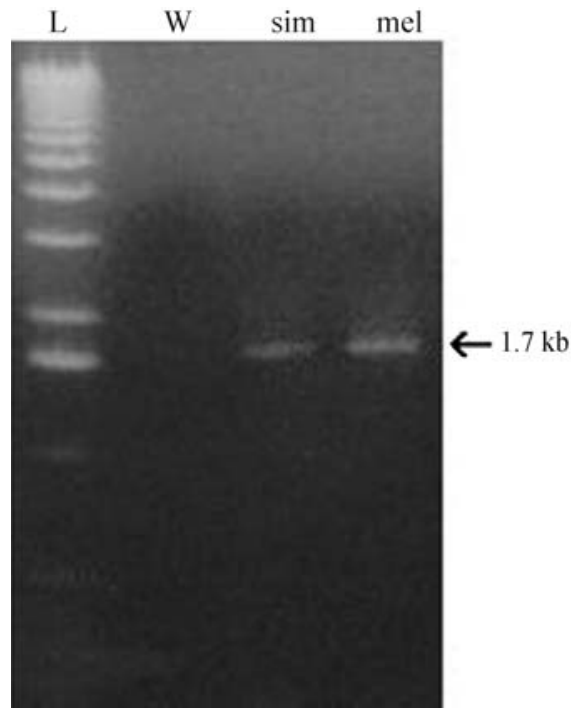

Figure 1 - The Bari-1 transposable element PCR products corresponding to the complete sequence in $D$. simulans and $D$. melanogaster strains visualized in 1\% agarose gel. Lanes are as follows: L, $1 \mathrm{~kb}$ DNA ladder size marker (GIBCO-BRL); W, water; sim, D. simulans C167.4; mel, D. melanogaster $\mathrm{IN}(1) \mathrm{AB}$.

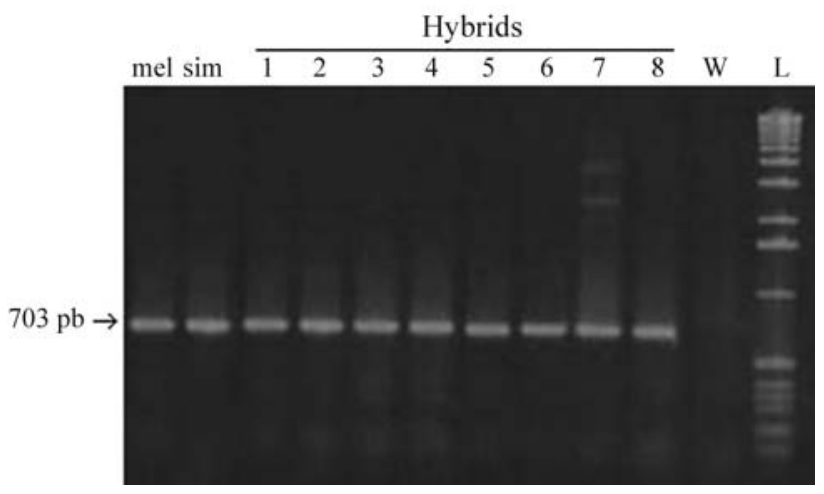

Figure 2 - The Bari- 1 transposable element PCR products corresponding to the internal segment with $703 \mathrm{bp}$ in D. simulans, D. melanogaster and hybrid strains visualized in $1 \%$ agarose gel. Lanes are as follows: mel, $D$. melanogaster $\mathrm{IN}(1) \mathrm{AB}$; sim, D. simulans $\mathrm{C} 167.4 ; 1-8$ : $\mathrm{SM}_{1}$ to $\mathrm{SM}_{8}$ hybrid strains; W, water; L, 1 kb DNA ladder size marker (GIBCO-BRL).

strains is shown in Figure 3. We can see that strain IN(1)AB presents the highest Bari-I copy number, as highlighting by the in tandem arrangement indicated by the thick $1.7 \mathrm{~kb}$ band, while strain C167.4 showed a smaller number of copies and an absence of the $1.7 \mathrm{~kb}$ band. These results are in agreement with those obtained by Caggese et al. (1995) regarding the distribution of the Bari-I element transposable in D. melanogaster and D. simulans. In our study, all hybrid strains showed some bands originating from one, or both, parental species. In addition, besides bands that were present in the parental $D$. simulans and D. melanogaster strains, the hybrid strain $\mathrm{SM}_{2}$ displayed a band originally not found in either of the parent strains, while strains $\mathrm{SM}_{3}$ and $\mathrm{SM}_{4}$ showed a band originally found in D. melanogaster but not

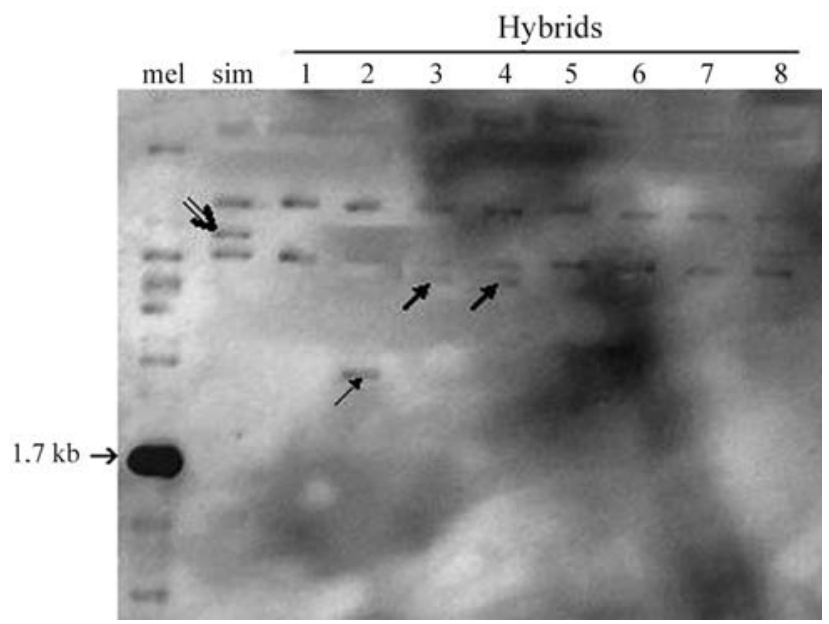

Figure 3 - Hybridization of 703 bp probe homologous to Bari-I internal sequence with genomic DNA of Drosophila melanogaster, D. simulans and hybrids strains after cleavage with Hind III restriction endonuclease. Lanes are as follows: mel, D. melanogaster IN(1)AB; sim, D. simulans C167.4; 1-8, $\mathrm{SM}_{1}$ to $\mathrm{SM}_{8}$, respectively. Thin arrow show band not found in D. melanogaster or D. simulans but present in $\mathrm{SM}_{2}$ hybrid strain. Thicker arrows show bands in $\mathrm{SM}_{3}$ and $\mathrm{SM}_{4}$ hybrid strains and in D. melanogaster but no in $D$. simulans. Double arrow shows band found in $D$. simulans but absent in $\mathrm{SM}_{1}$ to $\mathrm{SM}_{7}$ hybrid strains. 
in $D$. simulans. Furthermore, all the hybrid strains except $\mathrm{SM}_{8}$ showed the loss of one band initially present in $D$. simulans.

For the Brazilian D. melanogaster and D. simulans strains different patterns occurred for both species (Figure 4). In general, the Bari-I copy number was higher in $D$. melanogaster strains than in $D$. simulans strains and, disconsidering the in tandem array, the Bari-I copy number for $D$. melanogaster strains varied between three for strain SM to nine for strain (NH), while the Bari-I copy for $D$. simulans varied from one in strains $\mathrm{BG}$ and $\mathrm{NH}$ to seven in strain MN. There was no association between the Bari-I copy number and geographic location for the $D$. melanogaster strains but for the $D$. simulans strains the $\mathrm{MN}$ and GO strains from northern Brazil had higher copy numbers than the other strains from further south. This suggests a possible relationship between copy number and geographic localization, although more strains need to be studied to allow any firm conclusions about such relationships.

\section{Discussion}

The analysis of Bari I element copy number in $D$. melanogaster and $D$. simulans Brazilian populations are in agreement with Caggese et al. (1995) that showed a higher Bari-I copy number and the occurrence of a tandem arrangement in $D$. melanogaster not presented in $D$. simulans. These results are probably related to the fact that the $D$. melanogaster genome has many more insertion sites for many transposable elements than the D. simulans genome (Bièmont and Cizeron, 1999).

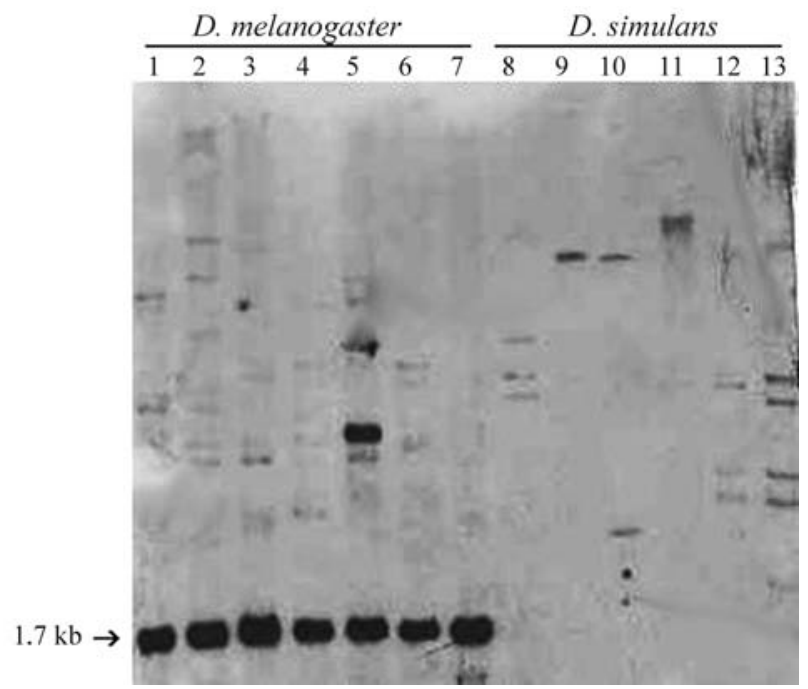

Figure 4 - Hybridization of 703 bp probe homologous to Bari-I internal sequence with genomic DNA of Drosophila melanogaster and D. simulans Brazilian populations after cleavage with Hind III restriction endonuclease. Lanes are as follows: 1-7: 1, D. melanogaster IN(1)AB; 2, TE (Teresina, PI); 3, SL (São Luís, MA); 4, SR (Santana do Riacho, MG); 5, NH (Novo Horizonte, SP); 6, MA (Maringá, PR); 7, SM (Santa Maria, RS); 8-13: D. simulans; 8, D. simulans C167.4; 9, BG (Bento Gonçalves, RS); 10, MA (Maringá, PR); 11, NH (Novo Horizonte, SP); 12, GO (Goiânia, GO); 13, MN (Manaus, AM).
One of the aims of our study was to investigate the possible transference of Bari-I sequences from $D$. melanogaster to the $D$. simulans genome that could have occurred during the establishment of the hybrid strains. In a previous study by one of the authors of the present paper the same hybrid strains were found to contain seven copies of the D. melanogaster copia retrotransposable element introgressed in $D$. simulans background genome (data not shown, unpublished results by C. R. Ceron). In the study described in the present paper, we observed introgression of Bari-I sequences, originally only found in $D$. melanogaster, into the hybrid strains, suggesting the concomitant introgression of these two TEs. In fact, the hybrid strains $\mathrm{SM}_{3}$ and $\mathrm{SM}_{4}$ presented Bari-I sequences from $D$. melanogaster (Figure 3, thick arrows), indicating that hybridization and introgression of the genetic material had occurred. Sawamura et al. (2000) also demonstrated introgression of genetic material carrying a complex of six sterility genes from the $D$. simulans into the $D$. melanogaster genome. Capy et al. (1998) has stated that although horizontal transfer is frequently invoked as a transfer mechanism, in the transfer of genetic material between sexually isolated species at least two mechanisms can operate: transfer by vectors and/or transfer by fertile hybrids retrocrossed with one of the parental species, our results supporting the latter mechanism.

As mentioned above, the Bari-I element has euchromatic and heterochromatic copies that are identical in size and sequence, which suggests that they are active elements in both portions of the genome (Caizzi et al., 1993). Although it was not possible to determine conclusively whether the transference of Bari-I to the D. simulans genome occurred by transposition or recombination in the hybrids, our data provides some hints. For example, the bands in the $\mathrm{SM}_{3}$ and $\mathrm{SM}_{4}$ hybrids corresponding to those normally present only in $D$. melanogaster were probably the result of introgression whereas the bands in these hybrids that were not found in D. simulans or in D. melanogaster could be due to transposition (as observed in the SM2 hybrid) or ancestral polymorphism present in some strains of one or both species. We also observed that in some hybrid strains $\left(\mathrm{SM}_{1}\right.$ to $\left.\mathrm{SM}_{7}\right)$ some of the copies that were initially presents in the D. simulans genome were deleted. In these cases, these sequences might have been deleted by recombination events or even by excision, although an alternative explanation is that only the DNA sequences of $D$. melanogaster, which did not harbor Bari-I insertions, were maintained within the hybrid genomes.

Our proposition of introgression of the Bari-I sequence from the $D$. melanogaster genome to the $D$. simulans genome is supported by work concerning the transposable elements common in D. melanogaster and the two related species D. simulans and Drosophila yakuba (Sanchez-Gracia et al., 2005). In nine out of the eleven families studied by Sanchez-Gracia et al. (2005) the divergence 
between $D$. melanogaster and $D$. simulans elements was much lower than that observed for nuclear genes, these workers properly interpreting this as an unexpectedly increased rate of horizontal transfer of transposable elements between these species and that variation of the divergence values across transposable element families suggest that the horizontal transfers might have occurred in multiple independent steps rather than being the result of a single episode of genomic admixture between two species. Even though we partially agree with Sanchez-Gracia et al. (2005), it is difficult to imagine that such an increased rate could be the result of horizontal transfer only. Our results suggest that hybridization may also have played a significant role in the evolutionary history of transposable elements in D. melanogaster and D. simulans.

\section{Acknowledgments}

The authors are grateful to Dr. Ruggero Caizzi for providing the Bari-I sequence cloned in pUC 8 vector. We also thank Dr. Luciane Almeida Madureira, Dr.Juliana P. de Castro, Dr. Milla A. Baffi and Dr. Ester Tartarotti for technical support. Thanks to Ms. Eliane N. Ohira for technical assistance. This work was supported by the Brazilian agencies FAPESP and CNPq. M.L. Jr. was the recipient of a fellowship from FAPESP (00/10690-4).

\section{References}

Bièmont C and Cizeron G (1999) Distribution of transposable elements in Drosophila species. Genetica 105:43-62.

Cagesse C, Pimpinelli S, Barsanti P and Caizzi R (1995) The distribution of the transposable element Bari-I in the Drosophila melanogaster and Drosophila simulans genomes. Genetica 96:269-283.

Caizzi R, Cagesse C and Pimpinelli S (1993) Bari-I a new transposon-like family in Drosophila melanogaster with a unique heterochromatic organization. Genetics 133:335-345.

Capy P, Bazin C, Higuet D and Langin T (1998) Dynamics and Evolution of Transposable Elements. Landes Bioscience, France, 197 pp.

Carracedo MC, Asenjo A and Casares P (1998) Inheritance mode of Drosophila simulans mating propensity with $D$. melanogaster males. The Journal of Heredity 89:101-104.
Crozatier M, Vaury C, Busseau I, Pélisson A and Bucheton A (1988) Structure and genomic organization of $I$ elements involved in I-R hybrid dysgenesis in Drosophila melanogaster. Nucleic Acids Res 16:9199-9213.

Davis AW, Roote J, Morley T, Sawamura K, Herrman S and Ashburner M (1996) Rescue of hybrid sterility in crosses between D. melanogaster and D. simulans. Nature 380:157159.

Di Nocera PP, Graziani F and Lavorgna G (1986) Genomic and structural organization of $D$. melanogaster $G$ elements. Nucleic Acid Res 14:675-691.

Jowett T (1986) Preparation of nucleic acids. In: Roberts DB (ed) Drosophila: A Practical Approach. IRL Press Oxford, pp 275-286.

Kamping A and van Delden W (1988) Hybridization between Drosophila melanogaster and D. simulans in nature. DIS 67:53.

Mensua JL and Perez M (1977) Hybridization in nature of Drosophila melanogaster and D. simulans. DIS 52:60.

O'Hare K and Rubin GM (1983) Structures of $P$ transposable elements and their sites of insertion and excision in the Drosophila melanogaster genome. Cell 34:25-35.

Rozenzweig B, Liao LW and Hirsh D (1983) Sequence of the $C$. elegans transposable element Tc1. Nucleic Acids Res 11:4201-4209.

Sánchez-Gracia A, Maside X and Charlesworth B (2005) High rate of horizontal transfer of transposable elements in Drosophila. Trends in Genetics 21:200-203.

Sawamura K, Davis AW and Wu C (2000) Genetic analysis of speciation by means of introgression into Drosophila melanogaster. PNAS 97 6:2652-2655.

Sperlich D (1962) Hybrids between Drosophila melanogaster and D. simulans in nature. DIS 36:118.

Streck RD, Macgaffey JE and Beckendorf SK (1986) The structure of hobo transposable elements and their insertion sites. EMBO J 5:3615-3623.

Surtevant AH (1920) Genetic studies on Drosophila simulans. I. Introduction. Hybrids with Drosophila melanogaster. Genetics 5:488-500.

Tracey ML, Pavlosky O and Green MM (1973) Hybridization of Drosophila melanogaster and D. simulans. DIS 50:77.

Vaury C, Bucheton A and Pélisson A (1989) The $\beta$-heterochromatic sequences flanking the I elements are themselves defective transposable elements. Chromosoma 98:215-224.

Associate Editor: Fábio de Melo Sene 